\title{
基层水利工程质量监督管理创新的思考
}

\author{
王力宏 \\ 内蒙古自治区赤峰市喀喇沁旗王爷府镇人民政府水利站 \\ DOI:10.32629/hwr.v3i1.1836
}

[摘 要] 随着当前社会经济的发展,人们对于基层水利工程质量的要求也在提升,在这当中,采用传统的监督管理方式已经很 难有效的满足人们的实际需求, 所以就需要加强对基层水利工程质量监督管理工作加强创新。对基层水利工程质量监督管理 和当前的社会发展相结合, 因此本文主要就对基层水利工程质量监督管理的创新工作加强中总是,以此来对监督管理质量工作 加强提升,确保对基层水利工程的发展有着很好的促进作用。

[关键词] 基层水利工程; 质量监督; 管理创新

\section{1 基层水利工程质量监督管理创新的重要性}

基层水利工程质量监督管理内容非常丰富, 对于实际的 技术要求也非常高, 所以在工作开展中, 就需要确保每一个 施工环节质量符合要求。基层水利工程质量监督管理工作的 主要内容就是实现对产品的合理设计以及进行方案设计的 编制和对产品质量的过程实现监督和管理, 并且对这当中所 涉及的相关管理体系和参数做好相应的检查以及监督。

并且还需要加强对基层水利工程施工当中的相关技术 应用以及操作的规范性加强检查, 按照审查的结果实施相应 的质量评估, 在这当中, 主要就是对工程资料加强管理以及 对工程实体做好管理等。比如, 基层水利工程在施工竣工环 节, 通常需要加强对工程建设质量加强有效的合适以及质量 的检查, 按照实际的结果对工程整体进行相应质量的评定, 并且在此基础上制定相应的质量报告。

因此可以看出, 基层水利工程质量监督管理工作有着非 常重要的作用, 对质量可以实现有效监测和规范, 确保基层 水利工程建设单位在对自身建设当中所存在的问题及时分 析, 并且将基层水利工程建设质量不断提升。所以, 在实际的 基层水利工程质量监督和管理当中, 需要能够按照时代的实 际发展需求和基层水利工程实施相关情况进行创新, 将基层 水利工程质量管理的传统方式进行摈弃, 因此, 只有加强创 新力度的提升, 对水利工程建设进行相应的监督和管理, 将 监督管理效率不断提升, 并且将其执行力有效提升, 确保能 够和时代的发展相符合。

\section{2 基层水利工程质量监督管理模式中存在的问题}

2.1 质量监督管理人员的素质较低

目前在基层水利工程建设中对于提供技术的要求非常 高, 基层水利施工的前期涉及的内容比较多, 包括基坑的勘 察、桩基以及地基等多个方面, 各个流程非常复杂, 因此对于 施工人员的要求非常高。但是目前在实际操作过程中, 相关 人员专业能力还需要进一步提高, 他们整体的能力不足, 同 时缺乏相应的培训与教育, 从而使得施工过程出现很多问题, 在应用中不能够明确图纸的相关意图, 也没有重点掌控施工 具体状况, 从而造成经济损失, 威胁到人们的健康。

\section{2 质量监督管理部门存在问题}

当前, 对于基层水利工程质量监督管理人员来讲, 在实 际的编制当中还缺少统一性, 对于质量监督管理部门来讲, 其组成部分主要由两个方面组成, 分别是独立的相关事业单 位管理部门和主管部门的事业单位, 这两个单位的工作性质 和公务员相似, 基本上都是由政府来实施拨款。另外, 对于一 些比较小的县城或者城镇来讲, 没有进行相应质量监督管理 部门的设置, 但是相对于我国水利部门所要求, 具有水利工 程的区域一定要能够实现对质量监督管理部门的完善, 以此 来确保水利工程能够有效实施, 然而对于一些区域因为相关 原因, 对质量监督管理部门的成立还有很大的难度, 尽管一 些区域按照相应的要求成立了质量监督管理部门, 但是其所 呈现出来的作用不是很大, 缺少比较专业的管理人员, 因此 对于这种情况在一定意义上对于基层水利工程的安全开展 也有很大的制约。

2.3 缺乏完善的法律法规

尽管当前我国已经颁布了相应的法律法规, 然而在一定 意义上还不是很完善, 所以, 相对于很多法律法规来讲, 主要 都是针对一些大型的水利工程项目, 相对于基层小型水利工 程项目所产生的制约性不是很强。一些政府单位对于基层水 利工程建设的相关制度和规范也没有针对性制定, 或者所制 定的规章制度起不到相应的约束作用。基层水利工程在建设 中主要还是针对一些小型的水利工程, 所以, 相关管理部门 所颁布的法律法规所产生的作用不是很大, 造成基层水利工 程在建设中缺少法律法规的约束, 对于基层水利工程的发展 也有一定的制约。

\section{4 质量监督管理方式较为落后}

随着当前社会经济的发展, 基层水利工程建设项目也在 不断增多, 这样就是的质量监督管理人员所承担的工作量也 在加大。现阶段, 对于基层水利工程质量监督和管理的现状 主要还是专业人员比较少, 并且工作量很大, 造成基层水利 工程监督管理浮于表面, 对其缺少深入性的监督和管理。并 且一些监督管理人员在对于建筑材料进行确认当中, 只是按 照生产厂家的资料进行作为判断依据, 在实际的水利工程施 
工建设当中, 按照相关施工单位所提供的资料对工程质量实 施评定, 造成基层水利工程有着很大的安全隐患存在。质量 监督管理人员往往只是按照自身的主观意识来对工程质量 实施判断, 对其缺少相应的考察, 这种传统以及落后的质量 监督管理模式和时代的发展不相符合, 因此就需要加强改革 和创新。

\section{3 基层水利工程质量监督管理创新措施}

3.1 加快完善质量监督相关法律法规

目前在社会快速发展的过程中, 在各行各业中都在投入 信息技术的使用, 所以在施工质量监督机制中使用信息化的 管理技术有着至关重要的作用。在水利工程施工的过程中对 实际施工的环境, 施工时所使用的工程材料进行严格的检查, 在检查原材料的过程中需要保证所使用的工程材料的质量, 杜绝在工程中施工的过程中出现偷工减料的情况, 除此之外, 在施工时如果出现了相关的质量问题就需要针对此种问题 来建立相关的预警机制, 以此保证能够在第一时间对出现的 质量问题给以解决。

3.2 提高质量监督管理部门的执行力

如今在水利项目建设过程中质量监督人员还需要进一 步提高, 在施工操作中需要相关人员严格的按照质量标准要 求进行操作, 但是因为施工队伍专业能力的限制, 从而使得 整体建设效果受到影响。目前施工企业没有高度重视水利施 工质量的监督控制, 在建设的过程中出现偷工减料的情况, 施工工序也没有严格按照要求进行, 从而都埋下了很多的安 全隐患, 直接影响到工程质量。对此质量监督人员必须要进 行优化控制, 在项目施工方案编制的过程中需要赋予质量监 督部门相应的权利和执行力, 能够在发现操作中发现问题及 时地采取措施进行解决, 从而更好的保证整体的质量, 也能 够更好地树立质量监督部门的威信。

3.3 加快质量监督方式方法的创新

第一, 强化集体监督管理制度的建立。对集体监督集体 不断完善, 通常, 因为个人监督会产生不公平以及客观的状 况, 但是对于集体监督就可以防止出现这种情况, 以此来是 的质量监督工作能够体现出相应的透明性。在对于基层水利 工程质量监督管理当中, 一定要能够保证监督管理人员和责 任监督管理人员都有相应的责任, 同时需要配备两名人员来 实施监督。第二, 加强相关设备的配备。智联监督管理部门
需要加强相关检测设备和仪器的配置, 对现场做好相应的检 查, 不能采用传统的方式进行检查, 需要采用回弹仪以及钢 筋扫描仪等设备对质量加强检查, 对相应的数据进行收集, 同时对数据做好处理和分析, 确保质量监督管理工作的科学 合理性。第三, 采用第三方检测。需要第三方检测企业和施 工企业没有相应的联系, 并且需要确保具有独立的法人, 单 位能够有能力提供专业的技术人员和仪器设备, 所呈现出来 的检测结果能够确保公平以及客观。

3.4 提高水利工程质量监督队伍建设水平

在对质量监督管理人员的配置当中, 不但需要确保专业 搭配的合理性, 还需要确保人员整体结构的科学合理性, 除 了需要采用一些经验丰富的质量监督人员, 还需要加强对新 人员的合理培训和培养, 在此基础上相关部门加强对相应质 量监督管理工作的内容进行教育培训, 对质量监督工作的经 验进行交流, 使得所有的质量监督人员都能够有效的参与到 工作当中, 以此来将其自身的业务能力和职业素养提升。相 对于质量监督管理人员来讲, 需要对质量监督管理工作的重 要性有效认识, 对相应法律法规加强学习, 使得其自身的业 务水平能够提升, 以此能够很好的实现质量监督工作的顺利 实施。

\section{4 结束语}

总之, 随着当前人们对于基层水利工程质量监督管理工 作的重视, 加强对基层水利工程质量监督管理的质量加强保 证, 是人们所重视的主要问题。因此本文主要就对其创新途 径加强分析, 以此将基层水利工程质量监督管理的有效性提 升, 确保质量科学合理。由此可以看出, 研究基层水利工程质 量监督管理工作的创新途径, 能够为今后基层水利工程质量 监督管理创新的发展奠定基础。

\section{[参考文献]}

[1]傅豪函.县级水利工程质量监督机构管理工作的创 新[J].河南水利与南水北调,2017(05):79-80.

[2]潘丽崴.简述小型水利工程建设的质量管理 [J].民营 科技,2016(04):90.

[3]吴涛.基层水利工程质量监督管理创新研究 [J].低碳 世界,2016(07):87-88.

[4]傅夏冰,陈智和.金华市加强水利工程质量监督的实 践与探索[J].中国水利,2013(08):34-36. 